\title{
The Future of Technology within an Aging Population
}

\author{
By Amy Doherty
}

T

he explosion of technological growth is changing the world and challenging everyone to imagine new implications for society. We often look at the smartphone we hold in our hand, with its ecosystem of apps and immediate access to information, as the norm. However, at any given point in time, technology will be exponentially better than it was yesterday. In technology, the only constant is the acceleration of change.

Whatever can be digitized has the opportunity to disrupt. More importantly, how we respond to enabling technologies, or innovations that power radical change, will have great implications for the evolution and sustainability of new markets and economies.

Technology accelerates through bonds with other technologies. For example, technological advances in transistors led to major accelerations in software development. Each technology influences the compounding growth of the other, resulting not in linear improvements but exponential acceleration. Similarly, cloud technologies and big data are bound together, propelling machine learning algorithms with massive data sets to create amazing advancements in how we manage information. With the overlay of technologies such as voice enablement, intelligent agents can enable new human capabilities.

The implications of exponential technology growth are enormous, touching on our health, mobility, work, passions, and even the length of our lives. We see fantastic improvements for a worldwide population that is growing older faster than any time in our history. A single technology advancement does not usually create a breakthrough innovation, but the collision of several technologies, powered by exponential trends, can disrupt global markets. Innovators can now draw from emerging technologies like artificial intelligence, biometric sensors, blockchain, embedded voice, process automation, and virtual reality to solve problems.

Exponential trends reduce costs and create a big shift in the ability to mobilize and scale when needed. Technology is a democratizing force, empowering people with creative ideas to bring their ingenuity to life by conquering production, marketing, and distribution challenges that have traditionally eliminated niche products from the marketplace. Falling prices and increased access to cloud technologies are driving software enablement and artificial intelligence to solve new problems.

While there is an explosion of technology and abundance, not everyone will experience this abundance. Innovators, change agents, and policy makers must identify and reject what I call discrimination-embedded technology. Algorithms created by complex machine-generated code are increasingly powering our information systems, but are they being trained with data sets representative of all generations? Consider advancements in more natural user interfaces like gestures and voice, wherein functionality is greatly influenced by the unintentional biases of the programmers and test groups. Automation to make our decision-making faster and easier can also introduce unintentional bias when it fails to include diverse populations. And it raises what is possibly the greatest question we can ask: are we applying our best analytical thinkers to our most impactful problems in society?

Organizations architecting next-generation technology infrastructures to deliver products and services have the potential to create great value for society by designing with all generations in mind. The customization of products and experiences made possible through leading-edge data analytics and infrastructures is infinite. Personalization is highly preferred by everyone, and for segments that are less familiar with technology, it offers innovators an enormous opportunity to enrich lives and create market share. As data, sensors, processors, and networks are optimized to their full potential by innovative software applications, we are moving toward true personalization, in which technology naturally considers the needs of individuals and how they interact with technology. This can be highly diverse among cultures, ages, perspectives, and experiences, which positions organizations that get it right to have a vast competitive advantage.

In collaboration, innovators and policy makers can explore how emerging technologies will collide to impact 


\title{
"Technology advancements will
} increasingly augment our health and routines, and humans worldwide are

\author{
on a trajectory to live longer, fuller lives \\ thanks to the application of code-based \\ machines of every size."
}

aging issues in an increasingly aging world. In many cases, surfacing these issues openly and transparently can fuel inclusive innovation. The future promises a fantastic opportunity of enablement, abundance, and personalization through accelerated technology efforts. As the number of older adults around the globe continues to climb, we owe them a parallel access to the future. Addressing those factors that lead to a "digital divide" of tech haves and havenots, such as cost, complexity, awareness, isolation, dialect, and distrust, can improve health and quality of life, and even save lives.

Technology advancements will increasingly augment our health and routines, and humans worldwide are on a trajectory to live longer, fuller lives thanks to the application of code-based machines of every size. Efforts are underway to ensure that those who do not have ready access to technology will not be disadvantaged.

In line with our social mission, AARP's greatest opportunity is disrupting the conversation related to aging to ensure fairness and bring technology-enabled innovation to market. As $\mathrm{CIO}$ I have the opportunity to meet with innovators and technology leaders from leading organizations and learn how they approach longevity. Everyone I meet has a story about an older family member or friend who is more connected, mobile, or healthy because of some recent tech-related offering. But I also hear concerns that organizations are not doing enough to ensure technology innovation impacts older generations at the same rate as younger generations.

The penetration of low-cost processors, human-centered apps, and low-friction user interfaces is allowing technology to augment our lives in amazing new ways. The best path forward for supplementing older adults with beneficial technology involves solving problems with personalization and humanity. Human-centered design considers the core challenges of how individuals want to interact with technology. Emerging technologies that are human and humane come with the promise to help augment the way we function and make us healthier, faster, sharper, mobile, and empowered to choose how we live as we age.

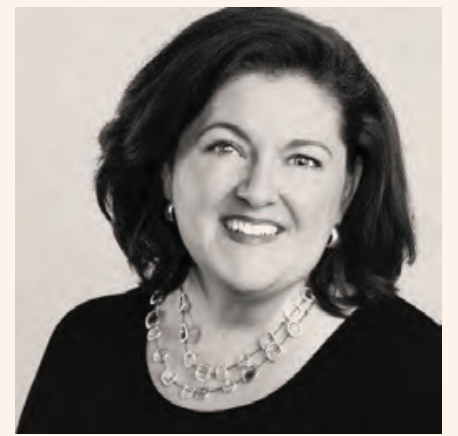

Amy Doherty SVP \& CIO, AARP 